\title{
A Van Atta reflector consisting of half-wave dipoles
}

\section{Appel-Hansen, Jørgen}

\section{Published in:}

I E E E Transactions on Antennas and Propagation

Publication date:

1966

Document Version

Publisher's PDF, also known as Version of record

Link back to DTU Orbit

Citation (APA):

Appel-Hansen, J. (1966). A Van Atta reflector consisting of half-wave dipoles. I E E E Transactions on Antennas and Propagation, 14(6), 694-700.

\section{General rights}

Copyright and moral rights for the publications made accessible in the public portal are retained by the authors and/or other copyright owners and it is a condition of accessing publications that users recognise and abide by the legal requirements associated with these rights.

- Users may download and print one copy of any publication from the public portal for the purpose of private study or research.

- You may not further distribute the material or use it for any profit-making activity or commercial gain

- You may freely distribute the URL identifying the publication in the public portal

If you believe that this document breaches copyright please contact us providing details, and we will remove access to the work immediately and investigate your claim 


\title{
A Van Atta Reflector Consisting of Half-Wave Dipoles
}

\author{
J. APPEL-HANSEN
}

\begin{abstract}
The reradiation pattern of a passive Van Atta reflector consisting of half-wave dipoles is investigated. The character of the reradiation pattern first is deduced by qualitative and physical considerations. Various types of array elements are considered and several geometrical configurations of these elements are outlined. Following this, an analysis is made of the reradiation pattern of a linear Van Atta array consisting of four equispaced half-wave dipoles. The general form of the reradiation pattern is studied analytically. The influence of scattering and coupling is determined and the dependence of the reradiation pattern on the length of the transmission lines interconnecting the dipoles is investigated. The optimum length of the transmission lines is determined. The results, found by the analysis of the reradiation pattern, are verified experimentally. The experimental reflector consists of slot-fed dipoles with open-ended terminations. Line stretchers are inserted into the transmission lines.
\end{abstract}

\section{INTRODUCTION}

$\mathrm{F}$ YOR MANY applications, such as echo enhancement and satellite communication, reflectors are needed which have a maximum of reradiation back in the direction of arrival of an incident wave. In 1955, Van Atta [1] proposed a reflector with this property. The reflector consists of antenna elements properly arranged and connected by transmission lines so that the signals received (or absorbed) from an incident wave by the antennas are reradiated in phase back in the direction of incidence, for all angles of incidence. This principle of operation will be called the Van Atta principle and a reflector operating in accordance with the Van Atta principle will be called, in agreement with general convention, a Van Atta array or a Van Atta reflector.

Since Van Atta proposed his reflector, several papers have suggested the application of the reflector in many communication systems [1]-[13]. A survey of previous papers dealing with Van Atta reflectors is also published in this issue of these Transactions [14]. Usually, only the Van Atta principle is considered and scattering by, and coupling between, the antennas are neglected. However, in the reports and papers mentioned subsequently, one or both of these effects are considered.

Sharp [2] made an experimental investigation of the back-reradiation, i.e., the reradiated energy back in the direction of incidence, from a planar reflector consisting of 16 dipoles. At different frequencies the back-reradiation was measured as a function of the angle of incidence. It was observed that at $3000 \mathrm{Mc} / \mathrm{s}$ the back-

Manuscript received March 3, 1966; revised July 5, 1966. This research was sponsored in part by the Air Force Cambridge Research Laboratories, under Contract AF 61(052)-794 through the European Office of Aerospace Research, U. S. Air Force.

The author is with the Laboratory of Electromagnetic Theory, Technical University of Denmark, Lyngby, Denmark reradiation, as a function of the angle of incidence, had a small minimum at normal incidence. This was not in accordance with the theory presented in the report. It was suggested that the minimum was due to scattering by the dipoles but a further investigation was not made.

In a discussion of a technique for amplitude modulation of a Van Atta radar reflector, Bauer [5] considered the back-reradiation and took into account scattering by the antennas. However, his considerations are only valid if the field due to scattering is of the same order of magnitude as the field reradiated due to the interconnections between the antennas. Furthermore, Bauer's expression for the back-reradiation is incorrect. This is shown in Appendix I of the present paper.

Østfeldt [15] considered a linear reflector consisting of half-wave dipoles. He derived an equivalent circuit for the dipoles and the transmission lines. For some rather arbitrarily chosen values of the spacing between the dipoles, the length and the characteristic impedance of the transmission lines, he calculated the reradiation pattern of the reflector. The patterns have a form which indicates that the reradiation pattern of a Van Atta reflector does not always have a maximum back in the direction of incidence. Neither a further analysis nor an explanation of this behavior was given.

Hansen [16] has mentioned that the Van Atta reflector "is the discrete analog of the corner reflector, and has similar interference lobes due to interaction of reradiated and scattered fields." Further details about the interference lobes were not given.

From the above discussion it appears that no general treatment of the influence of scattering and coupling on the reradiating properties of the reflector has been given. It is convenient at this point to state that by scattering we mean the following interaction effect between a dipole and the incident wave: energy is taken from the incident wave and reradiated into all directions about the dipole [17]. Another interaction effect between a dipole and the incident wave is that considered in the preceding description of the Van Atta principle: energy is taken (absorbed, received) from the incident wave, transmitted through the transmission line to the connected dipole, and there reradiated. By coupling we mean the interaction between the dipoles. Therefore, when we say that coupling is neglected, we mean that we do not take in to account the fields from the currents which the dipoles induce in each other.

It is the purpose of this paper to present a theoretical, experimental, and numerical investigation of the influ- 
ence of scattering and coupling on the reradiation pattern of a linear Van Atta reflector consisting of four halfwave dipoles. The four dipoles may be considered as two pairs of dipoles in an arbitrary Van Atta reflector and, because of this, it is possible to derive similar results for reflectors with more than four elements.

\section{Theory}

\section{A. The Van Atta Principle}

The Van Atta array consists of a number of antenna elements arranged in pairs. The elements in each pair are placed symmetrically about a conveniently selected center, which is common for all pairs. Furthermore, transmission lines of equal length connect the elements of each pair. An element may be placed at the center of the array and short circuited by a transmission line with a length equal to half the length of the other lines. However, without loss of generality, we will suppose in what follows that there is no such element at the center.

Due to the requirement on the location of the antenna elements, it is natural to propose linear, planar, circular, cylindrical, and spherical configurations of the antennas. Hansen [6] has proposed other geometrical configurations such as the tetrahedron, the cube, or the rectangular box with an array on each face. Furthermore, several arrays may be arranged on a cylindrical or a spherical surface. Hansen [6] has particularly discussed planar and cylindrical arrays. Davies [10], who has investigated the circular Van Atta array, has mentioned the possibility of building up a circular array from a number of arcs of a circle. Each arc consists of a linear array. An acoustic reflector consisting of two planar arrays has been experimentally investigated by Walther [8].

When a reflector is composed of a number of Van Atta arrays, it is important to take into account the interaction between the fields from the different arrays to ensure that they do not cancel each other. One could imagine that this condition might lead to reflectors composed of a number of arrays which do not constitute a simple geometrical body.

Applications of a Van Atta array are limited by the frequency band, polarization properties, and field pattern of the antenna elements.

Most often half-wave dipoles are chosen as antenna elements in a Van Atta array. They have been selected because of their omnidirectional pattern which gives the array broad-angle coverage. The limitation due to the polarization properties of the dipoles may be lessened by using crossed dipoles.

If the frequency band for operation of the half-wave dipoles is not sufficiently broad, wide-band radiators such as spiral antennas or conical helix antennas may be used. Furthermore, these antennas overcome the limitations of polarization sensitivity since they are circularly polarized. However, their directivity may in some cases limit the applications.
Broad-angle coverage, greater than that obtained by the wide-band antennas mentioned previously, may be obtained by using pyramidal horns as antenna elements. The coupling in an array consisting of horns will probably be less than that in an array consisting of halfwave dipoles. However, a reflector consisting of horns will have smaller angular coverage than a reflector consisting of half-wave dipoles.

In order to make a basic theoretical and experimental investigation of a Van Atta reflector, we will deal with a linear array consisting of four half-wave dipoles. Larsen [18] has shown how the investigation may be extended to an arbitrary configuration of half-wave dipoles. An extension of the investigation to other antenna elements would be of interest but will not be attempted in this paper.

Figure 1 shows the linear Van Atta reflector consisting of four parallel half-wave dipoles. The dipoles are arranged in two pairs. Let us neglect coupling between the dipoles and consider what happens when a plane wave is incident from an arbitrary direction. Each dipole is influenced by the incident wave and an excitation caused by the connected transmission line. This excitation is due to the fact that each dipole absorbs energy from the incident wave and this energy is transmitted to the connected dipole. Thus, we divide the current distribution generated on the dipoles into two parts. The first part is the current induced in each dipole by the incident wave. The second part is the current due to the connected dipole. Let the direction of propagation of the incident plane wave make an angle $\phi_{i}$ with respect to the plane of the reflector, i.e., the plane which contains the dipoles. From Fig. 2, we see that the fields from the first part of the above-mentioned currents are in phase in the direction $\pi-\phi_{i}$. These fields, which are due to scattering, will be called the scattered fields [17], and the in-phase addition at $\pi-\phi_{i}$ corresponds to a specular reflection. As mentioned in the Introduction, the scattered fields are usually neglected. Scattering, shown as follows, has a large influence. From Fig. 1 it is seen that the fields from the second part of the currents are in phase back in the direction of arrival of the incident wave. This is due to the equal lengths of the transmission lines. These fields are usually the only ones considered when the behavior of Van Atta reflectors is described, since it is these fields which produce a plane wave reradiated back in the direction of incidence.

In Appendix II, it is shown that for half-wave dipoles the field due to scattering is of the same magnitude as the field due to interconnections between the dipoles. Because of this fact, the reflector will have beams of equal magnitude, one in the specular direction and one back in the direction of incidence. However, due to the interference between the two fields the beams will of ten deviate from these directions. A case of particular interest occurs at normal incidence. Here, the two fields both 


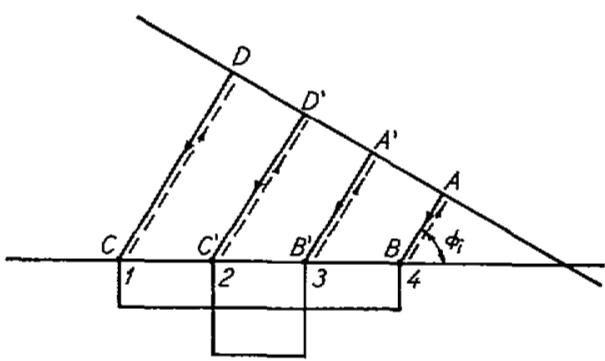

Fig. 1. The Van Atta principle. The paths $A B C D$ and $A^{\prime} B^{\prime} C^{\prime} D^{\prime}$ are equal.

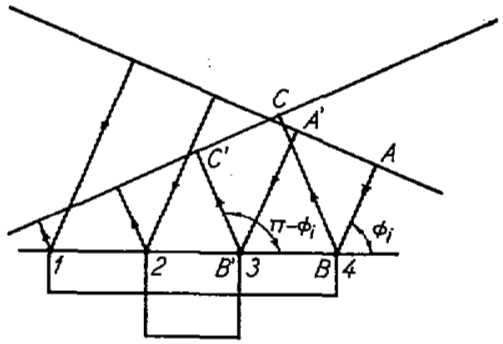

Fig. 2. The specular reflection. The paths $A B C$ and $A^{\prime} B^{\prime} C^{\prime}$ are equal.

have their beams back in the direction of incidence. Therefore, we may expect that for some length of the transmission lines the two fields have opposite phases and cancel each other. On the other hand, for some length the two fields are in phase and we have maximum reradiation.

In the foregoing we have neglected coupling. However, when the spacing between the dipoles is smaller than one wavelength, which may be the case for a Van Atta array, coupling has a considerable influence. Therefore, we may expect that coupling increases or decreases the reradiation, depending on the distance between the dipoles, the length of the transmission lines, and the angle of incidence.

From the above-mentioned facts, it appears that if the leng th of the transmission lines is chosen arbitrarily, we cannot expect a maximum reradiation back in the direction of incidence. However, it will be shown later that considerable back-reradiation will be achieved if the appropriate length of the lines is used. Therefore, this must be taken into account if we wish to use a reflector consisting of half-wave dipoles.

\section{B. The Reradiated Field}

For the reflector shown in Fig. 1, we will now deduce an expression for the reradiated field. Let us suppose that an incident plane wave induces the total currents $I_{1}, I_{2}, I_{3}$, and $I_{4}$ in the four dipoles numbered $1,2,3$, and 4 , respectively. Using equivalent circuits for the dipoles and T-circuits for the transmission lines, $\emptyset$ stfeldt [15] has shown that these four currents are determined by

$$
\begin{gathered}
\left(Z_{A n}+i Z_{0} \cot \beta l\right) I_{1}+Z_{12} I_{2}+Z_{12} I_{3} \\
+\left(Z_{14}+i \frac{Z_{0}}{\sin \beta l}\right) I_{4}=V_{1} \\
Z_{12} I_{1}+\left(Z_{A n}+i Z_{0} \cot \beta l\right) I_{2} \\
\quad+\left(Z_{12}+i \frac{Z_{0}}{\sin \beta l}\right) I_{3}+Z_{13} I_{4}=V_{2} \\
Z_{13} I_{1}+\left(Z_{12}+i \frac{Z_{0}}{\sin \beta l}\right) I_{2} \\
\quad+\left(Z_{A n}+i Z_{0} \cot \beta l\right) I_{3}+Z_{12} I_{4}=V_{3} \\
\left(Z_{14}+i \frac{Z_{0}}{\sin \beta l}\right) I_{1}+Z_{13} I_{2}+Z_{12} I_{3} \\
\quad+\left(Z_{A n}+i Z_{0} \cot \beta l\right) I_{4}=V_{4},
\end{gathered}
$$

where $V_{1}$ to $V_{4}$ are the free-space open-circuit voltages induced by the plane wave in dipoles 1 to 4, respectively. $l, Z_{0}$, and $\beta$ are the length, characteristic impedance, and propagation constant, respectively, of the transmission lines, which are assumed to be lossless. The time factor is $e^{-i \omega t} . Z_{A n}$ is the dipole impedance. $Z_{12}, Z_{13}$, and $Z_{14}$ denote the mutual impedances between half-wave dipoles with an interspacing of $d, 2 d$, and $3 d$, respectively. Due to the equispacing $d$ between the dipoles, a knowledge of $Z_{12}, Z_{13}$, and $Z_{14}$ provides the mutual impedance between any two dipoles if we neglect the presence of the remaining dipoles. Thus, in the numerical calculations described below, we use the values of the mutual impedances based upon the induced-EMF method. The dipoles are assumed to be infinitely thin and to have sinusoidal current distributions. It should be pointed out that the equivalent circuit used for the derivation of (1) to (4) is valid only under the assumption of sinusoidal current distribution $[17]$.

In order to clarify the preceding physical and qualitative considerations, we will consider in detail the case in which the dipoles are matched to the transmission lines, i.e., $Z_{A n}=R_{A n}=Z_{0}$, where $R_{A n}=73 \mathrm{ohms}$ is the resistance of a half-wave dipole. In these circumstances it is convenient to consider the transmission lines as phase-shifters (see Fig. 3).

From considerations of the equivalent circuit it appears that $I_{1}$ is the sum of the four currents $I_{11}, I_{12}, I_{13}$, and $I_{14}$, where

$$
I_{11}=\frac{V_{1}}{2 R_{A n}}
$$

due to $V_{1}$ on dipole 1 .

$$
I_{12}=-\frac{V_{4}}{2 R_{A n}} e^{i \beta l}
$$

due to $V_{4}$ on dipole 4 , which, through the transmission line, generates a current in dipole 1 . 


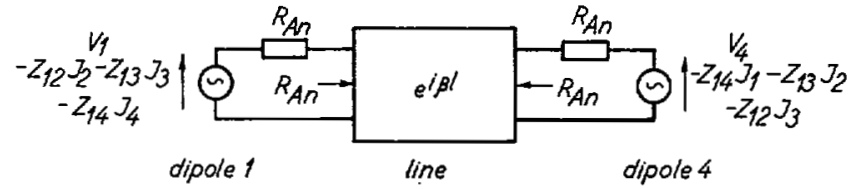

Fig. 3. Equivalent circuit for dipoles 1 and 4 .

$$
I_{13}=\frac{-Z_{12} I_{2}-Z_{13} I_{3}-Z_{14} I_{4}}{2 R_{A n}}
$$

due to coupling between dipole 1 and dipoles 2,3 , and 4 .

$$
I_{14}=-\frac{-Z_{14} I_{1}-Z_{13} I_{2}-Z_{12} I_{3}}{2 R_{A n}} e^{i \beta l}
$$

due to coupling between dipole 4 and dipoles 1, 2, and 3 . $I_{11}$ is associated with the scattering of dipole 1 . This is the current which, together with the corresponding currents from the other dipoles, produces specular reflection. $I_{12}$ is associated with the excitation from the transmission line. This is the current which, together with the corresponding currents from the other dipoles, produces a maximum of reradiation back in the direction of incidence. It appears that $I_{11}$ has the same magnitude as $I_{12}$. Furthermore, $I_{13}$ and $I_{14}$ are not negligible in comparison with $I_{11}$ and $I_{12}$ when the mutual impedances are of the same order of magnitude as $R_{A n}$. This is the case for a Van Atta reflector, where the spacing between the antennas is chosen to be less than one wavelength.

By using the relation $I_{1}=I_{11}+I_{12}+I_{13}+I_{14}$ and the corresponding relations for $I_{2}, I_{3}$, and $I_{4}$, we obtain

$$
\begin{aligned}
\left(2 R_{A n}\right. & \left.-Z_{14} e^{i \beta l}\right) I_{1}+\left(Z_{12}-Z_{13} e^{i \beta l}\right) I_{2} \\
& +\left(Z_{13}-Z_{12} e^{i \beta l}\right) I_{3}+Z_{14} I_{4}=V_{1}-V_{4} e^{i \beta l} \\
\left(Z_{12}-\right. & \left.Z_{13} e^{i \beta l}\right) I_{1}+\left(2 R_{A n}-Z_{12} e^{i \beta l}\right) I_{2}+Z_{12} I_{3} \\
& +\left(Z_{13}-Z_{12} e^{i \beta l}\right) I_{4}=V_{2}-V_{3} e^{i \beta l} \\
\left(Z_{13}-\right. & \left.Z_{12} e^{i \beta l}\right) I_{1}+Z_{12} I_{2}+\left(2 R_{A n}-Z_{12} e^{i \beta l}\right) I_{3} \\
& +\left(Z_{12}-Z_{13} e^{i \beta l}\right) I_{4}=V_{3}-V_{2} e^{i \beta l} \\
Z_{14} I_{1}+ & \left(Z_{13}-Z_{12} e^{i \beta l}\right) I_{2}+\left(Z_{12}-Z_{13} e^{i \beta l}\right) I_{3} \\
& +\left(2 R_{A n}-Z_{14} e^{i \beta l}\right) I_{4}=V_{4}-V_{1} e^{i \beta l},
\end{aligned}
$$

which is a special case of (1) to (4).

From (5) to (8) the currents in the dipoles may be calculated. Following this, by the superposition principle the far-field reradiation pattern of the array may be found (see next section).

\section{The General Form of the Reradiation Pattern}

In order to study the general form of the reradiation pattern we neglect coupling. Suppose that the direction of propagation of the incident wave is in the plane normal to the dipoles and makes an angle $\phi_{i}$ with respect to the plane of the reflector. Let $V_{t}$ be the free-space open-circuit voltage induced in a reference antenna placed at the center of the reflector. Then expressions for $V_{1}$ to $V_{4}$ may be easily obtained and $I_{1}$ to $I_{4}$ found from (5) to (8). Using the field pattern for a half-wave dipole [19] and the superposition principle, we obtain for the electric far-field intensity at a point in the plane normal to the dipoles, at a distance $r$ from the reference antenna in a direction making an angle $\phi_{u}$ with respect to the plane of the reflector.

$$
E=-\frac{i \xi}{\pi} \frac{e^{i k r}}{r} \frac{V_{t}}{R_{A n}} \cdot g
$$

where

$$
\begin{aligned}
g= & \cos 2\left(x_{i}+x_{u}\right) \cos \left(x_{i}+x_{u}\right) \\
& -\cos 2\left(x_{i}-x_{u}\right) \cos \left(x_{i}-x_{u}\right) e^{i \beta l} \\
x_{i}= & \frac{1}{2} k d \cos \phi_{i} \\
x_{u}= & \frac{1}{2} k d \cos \phi_{u} .
\end{aligned}
$$

$\xi$ and $k$ are the free-space characteristic impedance and propagation constant, respectively. A generalization of (9) which includes arbitrary directions of incidence, arbitrary polarization, and the entire reradiation pattern is easily made by using the general expression for the effective length of the dipoles and the field pattern of the dipoles [14].

From the absolute value of $g$ we find that the reradiation pattern is symmetrical about the normal to the plane containing the dipoles, the reradiation depends on the length of the transmission lines, and maximum reradiation often is not back in the direction of incidence. These results are in accordance with the physical and qualitative considerations. In the case of normal incidence, maximum reradiation is obtained for $l=0.50 \lambda$ $+p \lambda$, and there will be no reradiation for $l=1 \lambda+p \lambda$, where $\lambda$ is the wavelength and $p$ an integer or zero. In particular, for $d=0.50 \lambda$ and $l=1 \lambda+p \lambda$ we always have a maximum at $\phi_{u}=0^{\circ}$, and we have a maximum in the direction determined by

$$
\cos x_{i b}=\sqrt{\frac{9 \sin 3 x_{i}-\sin x_{i}}{12 \cos 3 x_{i}}},
$$

if we can find a real value of $\phi_{u}$ from (12) and (14). On account of symmetry we only consider $\phi_{i}$ and $\phi_{u}$ in the interval $0^{\circ}$ to $90^{\circ}$. For $d=0.50 \lambda$ and $l=1 \lambda+p \lambda$, Fig. 4 shows $\phi_{u_{\max }}$, the direction of the greater maximum, as a function of $\phi_{i}$. We see that there is often a large deviation between $\phi_{u_{\max }}$ and $\phi_{i}$. In Fig. 5, some reradiation patterns are shown for $d=0.50 \lambda, l=1 \lambda+p \lambda$, and $\phi_{i}=0^{\circ}$, $30^{\circ}, 60^{\circ}$, and $90^{\circ}$. From these patterns we notice that coupling does not influence the principal form of the pattern; it appears that coupling may increase or decrease the reradiation, but usually by not more than 50 percent. A further analysis [20] shows that, except for $l=0.50 \lambda+p 0.50 \lambda$, coupling causes the reradiation pattern to be asymmetrical about the normal to the plane containing the dipoles. 


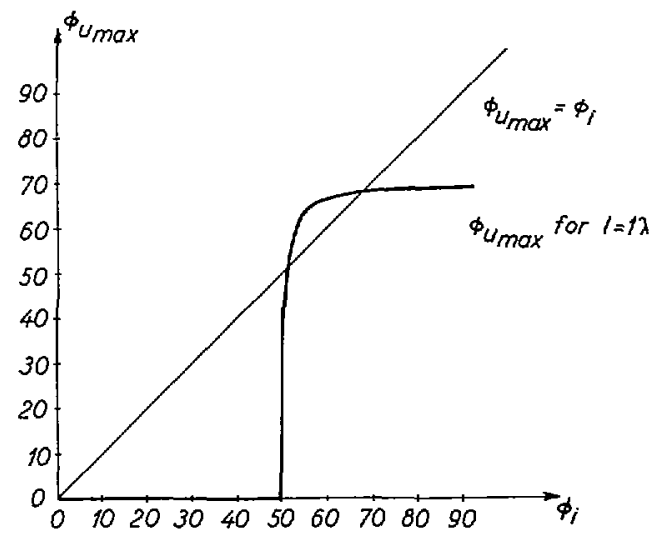

Fig. 4. $\phi_{u_{\text {max }}}$ as a function of $\phi_{i}$ for $l=1 \lambda+p \lambda$.

\section{The Optimum Transmission Line Length}

When $d>\lambda / 8$ and coupling is neglected, it has been found that the back-reradiation, as a function of the angle of incidence, has its largest minimum value for $l=0.25 \lambda+p 0.50 \lambda$. Coupling changes this result. For $d=0.50 \lambda$ numerical calculations have shown that the back-reradiation, as a function of the angle of incidence, has its largest minimum value for $l=0.64 \lambda+p \lambda$ (see Fig. 6). In general, for $d>0.50 \lambda$ and $l=0.25 \lambda+p 0.50 \lambda$, the reflector will have a back-reradiation which is not more than 50 percent below that obtained if coupling were not present. For $d<0.50 \lambda$ coupling decreases the reradiation, often by much more than 50 percent.

\section{EXPERIMENTAL WORK}

\section{A. Experimental Arrangement}

The experimental Van Atta reflector consists of four parallel equispaced slot-fed dipoles with open-ended terminations. Line stretchers are inserted in to the transmission lines connecting the dipoles. This is done in order to investigate the influence of the length of the lines on the reradiation properties of the dipoles. The measurements are performed at $3210 \mathrm{MIc} / \mathrm{s}$. The distance between the dipoles is $0.54 \lambda$.

The reflector is placed on a moveable pedestal in the middle of an anechoic box (see Fig. 7). The measurements are based on the principle of interference between two signals. When the pedestal is moved, the phase of the signal reradiated by the dipoles will vary. This signal, which is the one we wish to measure, interferes with a reference signal composed of reflections from the walls of the anechoic box and a signal introduced into the receiving system by the connection between the transmitting and receiving systems. By varying the amplitude and phase of the latter signal, it is possible to produce a reference signal of the same order of magnitude as the measured signal. This is done in order to have measurable interference.
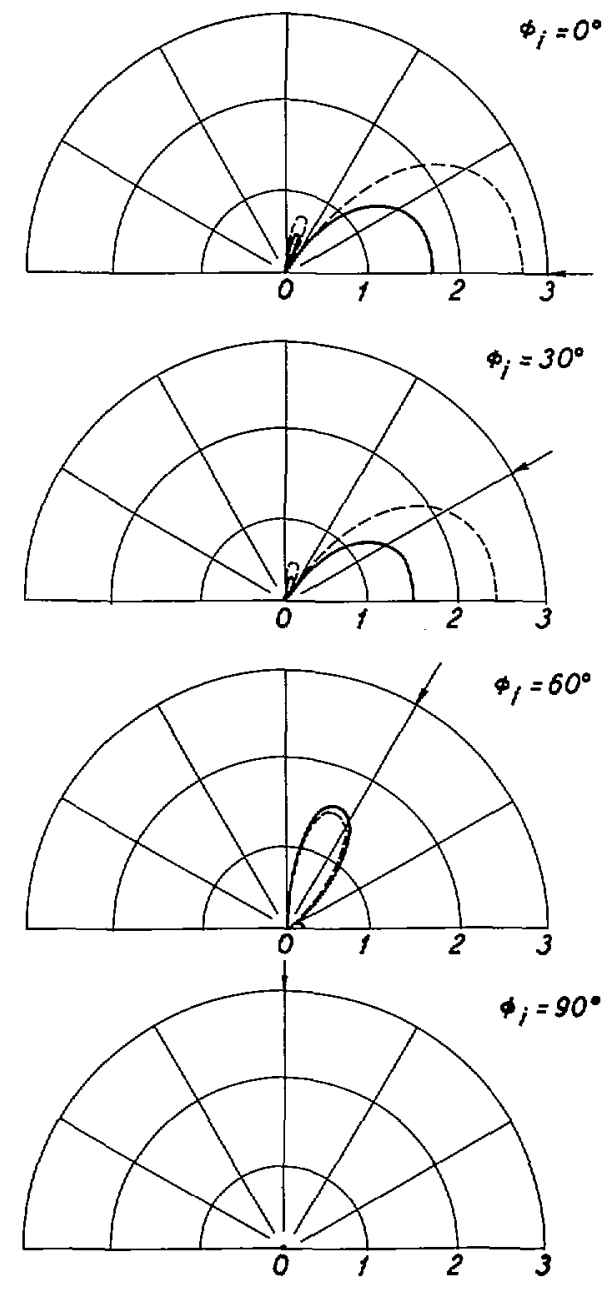

---coupling neglected

- coupling tokes into occount

Fig. 5. Reradiation patterns for $l=1 \lambda+p \lambda$.
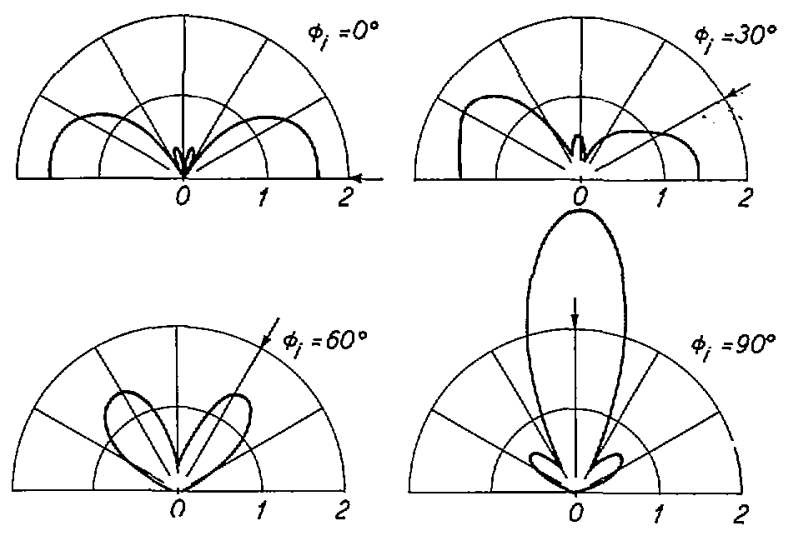

Fig. 6. Reradiation patterns for $l=0.64 \lambda+p \lambda$. Coupling is taken into account. 


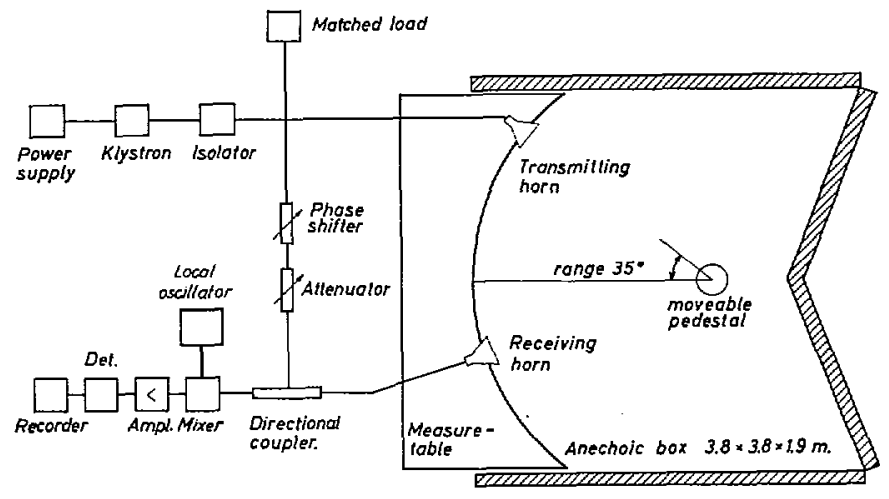

Fig. 7. Experimental arrangement.

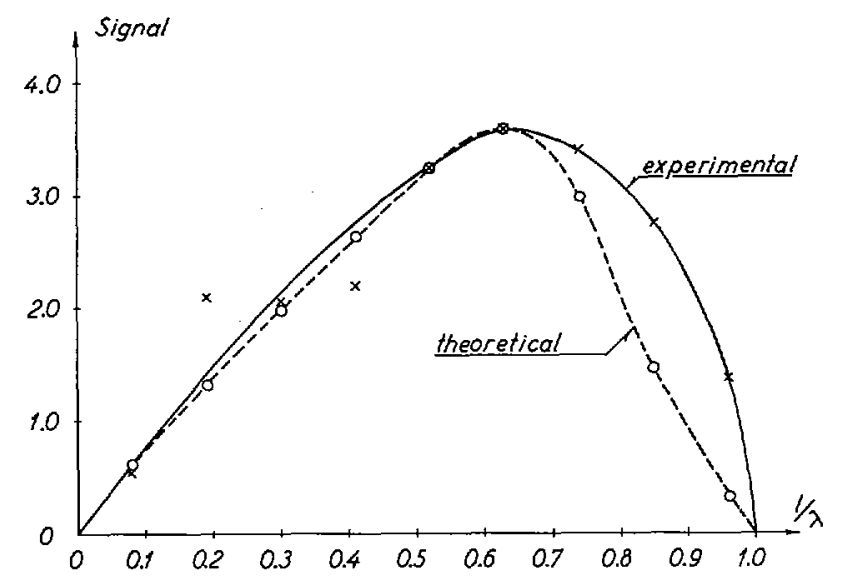

Fig. 8. Back-reradiation for normal incidence as a function of the length of the transmission lines.
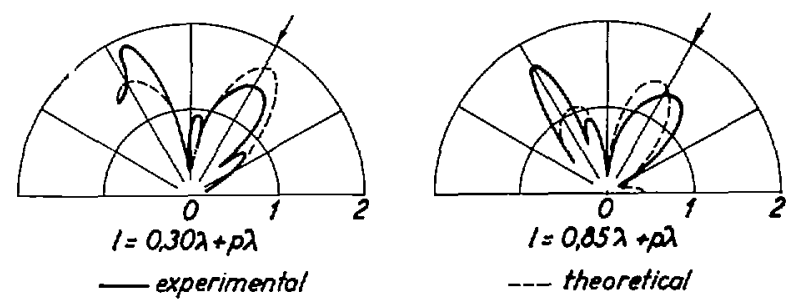

Fig. 9. Experimental reradiation patterns.

\section{B. Experimental Results}

In this paper, a small number of the experimental results obtained will be presented in order to confirm the following theoretical results.

1) The reflector reradiates in the specular direction to the same degree as it reradiates back in the direction of incidence.

2) The reradiation depends on the length of the transmission lines.
3) The maximum reradiation is not always back in the direction of arrival of an incident wave.

These results may be verified by considering Fig. 8 and Fig. 9. In Fig. 8 is shown, for normal incidence, the back-reradiation as a function of the length $l$ of the transmission lines. In order to compare the experimental and the theoretical results, a normalization is chosen so that there is agreement between the levels of the experimental and theoretical maxima. As expected from the theory, it appears that at normal incidence the magnitude of the back-reradiation is very dependent on $l$. When $l$ is about $1 \lambda+p \lambda$, the back-reradiation is negligible.

Figure 9 shows the reradiation patterns for $\phi_{i}=60^{\circ}$ and $l=0.30 \lambda$ and $0.85 \lambda$. In accordance with theory it is seen that scattering causes a beam near the specular direction. The discrepancies between theory and experiment may be explained by discussion of the sources of etror [20].

\section{APPENDIX I \\ COMMENTS ON "TECHNique for AMPLITUDE Modulating a Van Atta Radar Reflector"}

L. H. Bauer [5] considers a reflector consisting of two parallel dipoles. A phase shifter is inserted into the transmission line in order to demonstrate that the array can be amplitude modulated simply by varying the phase shift. Considering the scattered field and the field due to the interconnection, it is found that the amplitude of the back-reradiated field is proportional to

$$
\cos \left(\frac{B+k l}{2}\right) \cos (k d \cos \theta)
$$

see (6) in Bauer's paper, where $B$ is the phase-shift, $l$ [the length of the transmission line, 2d] the distance between the two dipoles, $\theta$ is the angle between the direction of propagation of the incident wave and the plane in which the two dipoles are located, and $k$ is the propagation constant, which is taken to be the same for both the transmission line and free space. However, the above expression is incorrect because the transmission path from the antennas to the wavefront of the backreradiated wave is ignored. Furthermore, the sign must be changed in his (1) and (3) for the scattered field.

By using the method of Section II-B, it may readily be found that the back-reradiated field is proportional to

$$
1+\cos ^{2}(2 k d \cos \theta)-2 \cos (2 k d \cos \theta) \cos (k l+B) .
$$

From this it is seen that it is possible to vary the amplitude, but not always from a maximum value to zero as stated by Bauer. In fact, this is only possible when $2 k d \cos \theta=p \pi$, where $p$ is an integer or zero. 


\section{APPENDIX II}

\section{SCattered ANd Received Energies}

It will be demonstrated that the scattered energy and the energy delivered to the load (received, absorbed) by a matched antenna are equal, if the power scattered by the open-circuited antenna is much less than that scattered by the matched antenna. If this is assumed to hold, the echo area of the antenna is [22]

$$
\sigma=\frac{4 A G R_{A n}^{2}}{\left|Z_{A n}+Z_{L}\right|^{2}}
$$

where $Z_{A n}$ and $Z_{L}$ are the input and load impedances of the antenna, $R_{A n}=\operatorname{Re}\left(Z_{A n}\right), A$ is the effective area, and $G$ is the power gain.

If the antenna is matched, from (17) we get

$$
\sigma=A G
$$

By using the definitions of $\sigma, A$, and $G$, the scattered and received energies are found to be the same and equal to $A S_{i}$, where $S_{i}$ is the intensity of the incident field.

For a half-wave dipole, the back-scattering cross section is $0.2 \lambda^{2}$ when the dipole is matched [23], and $0.01 \lambda^{2}$ when the dipole is open circuited [24]. This shows that the considerations in this paper are valid when the antennas in the reflector are half-wave dipoles. Similar considerations hold for smaller dipoles.

\section{Conclusion}

It has been shown that a passive linear Van Atta reflector consisting of half-wave dipoles does not operate precisely as claimed in Van Atta's patent description, that is, it does not have a maximum of reradiation back in the direction of incidence for arbitrary angles of incidence. This is due to scattering by, and coupling between, the dipoles.

The following has been deduced by qualitative and physical considerations.

1) Due to scattering the reflector reradiates in the specular direction to the same degree as it reradiates back in the direction of incidence.

2) The reradiation depends on the length of the transmission lines.

3) There is not always a maximum of reradiation back in the direction of incidence.

These three results have been derived theoretically and have been verified experimentally.

Based on a numerical investigation, it has been found that if we wish to use a Van Atta reflector consisting of half-wave dipoles, we should choose the length of the transmission lines to be equal to about $0.25 \lambda+p 0.50 \lambda$ and the distance between the dipoles to be larger than $0.50 \lambda$.

\section{ACKNOWLEDGMENT}

The author is grateful to his colleagues at the Laboratory of Electromagnetic Theory, Technical University of Denmark, Lyngby, for many encouraging discussions which led to much of this paper. In particular, the author thanks T. Larsen, R. F. Millar, R. E. Kleinman, and $E$. Drag $\phi$ Nielsen for reading and commenting on the manuscript.

\section{REFERENCES}

[1] L. C. Van Atta, "Electromagnetic reflector," U. S. Patent 2908002 , Serial no. 514040 , October 1959.

[2] E. D. Sharp, "Properties of the Van Atta reflector," Rome Air Development Center, Rome, N. Y. Tech. Rept. RADC-TR-5853, ASTIA Doc. AD-148 684, April 1958.

[3] J. A. Fusca, "Compact refector has ECM potential," Aviation Week, vol. 70, pp. 66-69, January 5, 1959.

[4] E. D. Sharp and M. A. Diab, "Van Atta reflector array, IRE Trans. on Antennas and Propagation, vol. AP-8, pp. 436-438, Julv 1960.

[5] L. H. Bauer, "Technique for amplitude modulating a Van Atta radar reflector," Proc. IRE (Correspondence), vol. 49, pp. 634-635, March 1961.

[6] R. C. Hansen, "Communications satellites using arrays," Proc. IRE, vol. 49, pp. 1066-1074, June 1961.

[7] W. F. Bahret, "Technique for amplitude modulating a Van Atta radar reflector," Proc. IRE (Correspondence), vol. 49, p. 1692, November 1961.

[8] K. Walther, "Model experiments with acoustic Van Atta reflectors," J. A coust. Soc. Amer., vol. 34, pp. 665-674, May 1962.

[9] R. D. Wanselow, "A proposed high gain wide angle coverage, passive, modulated re-radiator," IRE Trans. on Antennas and Propagation (Communications), vol. AP-10, p. 785, November 1962.

[10] D. E. N. Davies, "Some properties of Van Atta arrays and the use of 2-way amplification in the delay paths," Proc. IEE, vol. 110 , pp. 507-512, March 1963.

[11] D. E. N. Davies et al., "Discussion on some properties of Van Atta arrays and the use of 2-way amplification in the delay paths," Proc. IEE, vol. 111, pp. 980-982, May 1964.

[12] S. N. Andre and D. J. Leonard, "An active retrodirective array for satellite communication," IEEE Trans. on Antennas and Propagation, vol. AP-12, pp. 181-186, March 1964.

[13] E. L. Gruenberg and C. M. Johnson, "Satellite communications relay system using a retrodirective space antenna," IEEE Trans. on Antennas and Propagation, vol. AP-12, pp. 215-223, March 1964.

[14] T. Larsen, "Reflector arrays," IEEE Trans. on Antennas and Propagation, this issue, pp. 689-693.

[15] M. H. Østfeldt, "Linear array as a passive reflector," M.Sc. thesis, Laboratory of Electromagnetic Theory, Technical University of Denmark, Lyngby, 1963 (in Danish).

[16] R. C. Hansen, "Preface to the special issue on active and adaptive antennas," IEEE Trans. on Antennas and Propagation, val. AP-12, pp. 140-141, March 1964.

[17] S. Silver, Microwave Antenna Theory and Design, vol. 12. New York: McGraw-Hill, MIT Radiation Lab. Ser., 1949, sec. 3.16, pp. $42-44$.

[18] T. Larsen, "A theoretical investigation of Van Atta arrays," Laboratory of Electromagnetic Theory, Technical University of Denmark, Lyngby, Sci. Rept. 1, Contract AF 61(052)-794, November 1964.

[19] S. Silver, Microwave Antenna Theory and Design, vol. 12. New York: McGraw-Hill, MIT Radiation Lab. Ser., 1949, sec. 3.16, p. 98.

[20] J. Appel-Hansen, "Linear Van Atta reflector consisting of four half-wave dipoles," Laboratory of Electromagnetic "Theory, Technical University of Denmark, Lyngby, Sci. Rept. 2, Contract AF 61(052)-794, November 1964.

[21] - "Experimental investigation of a linear Van Atta rerefector," Laboratory of Electromagnetic Theory, Technical University of Denmark, Lyngby, Sci. Rept. 3, Contract AF 61(052)-794, May 1965.

[22] R. F. Harrington, "Electromagnetic scattering by antennas," IEEE Trans. on Antennas and Propagation (Communications), vol AP-11, pp. 595-596, September 1963.

[23] Y. Y. Hu, "Back-scattering cross section of center-loaded cylindrical antenna," IRE Trans. on Antennas and Propagation, vol. AP-6, pp. 140-148, January 1958.

[24] R. F. Harrington, "Small resonant scatterers and their use for field measurements," IRE Trans. on Microwave Theory and Techniques, vol. MTT-10, pp. 165-174, May 1962. 